\title{
AN ELECTRON-TRACKING Compton TELESCOPE for A SURVEY of the DEEP UNIVERSE by MeV GAMMA- RAYS
}

\section{$\operatorname{AUTHOR(S):~}$}

Tanimori, T.; Kubo, H.; Takada, A.; Iwaki, S.;

Komura, S.; Kurosawa, S.; Matsuoka, Y.; ... Takemura, T.; Tomono, D.; Ueno, K.

\section{CITATION:}

Tanimori, T.... [et al]. AN ELECTRON-TRACKING Compton TELESCOPE for A SURVEY of the DEEP UNIVERSE by MeV GAMMA-RAYS. Astrophysical Journal 2015, 810(1): 28.

\section{ISSUE DATE:}

2015-09-01

URL:

http://hdl.handle.net/2433/230142

RIGHT:

(C) 2015. The American Astronomical Society. All rights reserved. 


\title{
AN ELECTRON-TRACKING COMPTON TELESCOPE FOR A SURVEY OF THE DEEP UNIVERSE BY MeV
} GAMMA-RAYS

\author{
T. Tanimori ${ }^{1,2}$, H. Kubo ${ }^{1}$, A. Takada ${ }^{1}$, S. Imaki ${ }^{1}$, S. Komura ${ }^{1}$, S. Kurosawa ${ }^{3}$, Y. Matsuoka ${ }^{1}$, K. Miuchi ${ }^{4}$, S. Miyamoto ${ }^{1}$, \\ T. Mizumoto ${ }^{1}$, Y. Mizumura ${ }^{1,2}$, K. Nakamura $^{1}$, S. Nakamura ${ }^{1}$, M. Oda ${ }^{1}$, J. D. Parker ${ }^{1}$, T. Sawano ${ }^{1}$, S. Sonoda ${ }^{1}$, \\ T. TAKEMURA ${ }^{1}$, D. TOMONO ${ }^{1}$, AND K. UENO ${ }^{1}$ \\ ${ }^{1}$ Department of Physics, Kyoto University, Kitashirakawa-Oiwakecho, Sakyo-ku, Kyoto 606-8502, Japan; tanimori@ cr.scphys.kyoto-u.ac.jp \\ ${ }^{2}$ Unit of Synergetic Studies for Space, Kyoto University, Kitashirakawa-Oiwakecho, Sakyo-ku, Kyoto 606-8502, Japan \\ ${ }^{3}$ Institute of Materials Research, Tohoku University, 980-8577 Sendai, Japan \\ ${ }^{4}$ Department of Physics, Kobe University, 658-8501 Kobe, Japan \\ Received 2014 November 26; accepted 2015 July 13; published 2015 August 26
}

\begin{abstract}
Photon imaging for $\mathrm{MeV}$ gammas has serious difficulties due to huge backgrounds and unclearness in images, which originate from incompleteness in determining the physical parameters of Compton scattering in detection, e.g., lack of the directional information of the recoil electrons. The recent major mission/instrument in the $\mathrm{MeV}$ band, Compton Gamma Ray Observatory/COMPTEL, which was Compton Camera (CC), detected a mere 30 persistent sources. It is in stark contrast with the $\sim 2000$ sources in the $\mathrm{GeV}$ band. Here we report the performance of an Electron-Tracking Compton Camera (ETCC), and prove that it has a good potential to break through this stagnation in MeV gamma-ray astronomy. The ETCC provides all the parameters of Compton-scattering by measuring 3D recoil electron tracks; then the Scatter Plane Deviation (SPD) lost in CCs is recovered. The energy loss rate $(d E / d x)$, which CCs cannot measure, is also obtained, and is found to be helpful to reduce the background under conditions similar to those in space. Accordingly, the significance in gamma detection is improved severalfold. On the other hand, SPD is essential to determine the point-spread function (PSF) quantitatively. The SPD resolution is improved close to the theoretical limit for multiple scattering of recoil electrons. With such a well-determined PSF, we demonstrate for the first time that it is possible to provide reliable sensitivity in Compton imaging without utilizing an optimization algorithm. As such, this study highlights the fundamental weak-points of CCs. In contrast we demonstrate the possibility of ETCC reaching the sensitivity below $1 \times 10^{-12} \mathrm{erg} \mathrm{cm}^{-2} \mathrm{~s}^{-1}$ at $1 \mathrm{MeV}$.
\end{abstract}

Key words: gamma-ray burst: general - instrumentation: detectors -

nuclear reactions, nucleosynthesis, abundances - supernovae: general - techniques: imaging spectroscopy

\section{INTRODUCTION}

$\mathrm{MeV}$ gamma-ray astronomy provides the unique opportunity to study supernovae ( $\mathrm{SNe}$ ) as fresh radio isotopes in $\mathrm{SNe}$ emit MeV gamma-rays (Matz et al. 1988; Chevalier 1992; Iyudin et al. 1994; Maeda et al. 2012; Churazov et al. 2014). Studies of active galactic nuclei and galaxies reveal the evolution of the early universe (Zhang \& Beacom 2004; Inoue et al. 2013). Also, gamma-ray bursts are a promising probe to catch the first star (Mészáros \& Rees 2010; Nakauchi et al. 2012). Especially, $\mathrm{SNe}$ are the most fascinating objects and are vigorously studied in all fields of astronomy. Nevertheless, there still remain many fundamental mysteries, such as the origin of SNe type-Ia and nucleosynthesis. Although the thermonuclear explosion of a single degenerate White Dwarf (SWD) has been believed to be the origin of $\mathrm{SNe}$ Ia and accordingly has been used as a distance standard in cosmology, a merger of two WDs (DWD) has been frequently pointed out as the more plausible origin (Hillebrandt \& Niemeyer 2000). Recently, the importance of the observations in $\mathrm{MeV}$ gammas to conclusively determine the origin was remarked (Summa et al. 2013), considering that $\mathrm{MeV}$ gammas are the unique probe that are directly emitted from the exploding or merging regions. They pointed out that a delayed peaking time (80 days, compared to 50 days for SWDs) appears in DWDs due to its higher total mass, in contrast to the predicted model-independent peak time of 20 days from optical observations. Most of those sources are expected to be faint, because the number of the detected gammas is proportional to the cubic of the distance to the source. Each detection will contain a substantial amount of uncertainties, such as, fluctuations of ${ }^{56} \mathrm{Ni}$ production $( \pm 20 \%)$ and viewing angles (K. Maeda 2014, private communication). We then estimate that detection of $\sim 100 \mathrm{SNe}$, each of which should have $>5 \sigma$ significance in 10 days of observation, is required to obtain the statistically robust confirmation of these features. Given the optical results of $\sim 15$ and $\sim 50$ /year SNe Ia and collapsars (SNe Ib, c, and II), respectively, within $60 \mathrm{Mpc}$ (Maoz \& Mannucci 2012, K. Maeda 2014, private communication), an $\mathrm{MeV}$ instrument with a sensitivity of $\sim 10^{-12} \mathrm{erg} \mathrm{cm}^{-2} \mathrm{~s}^{-1}$ for $10^{6} \mathrm{~s}$ is needed to catch gammas of these $\mathrm{SNe}$ with the above-mentioned significance. Thus, to push such a new field in MeV-gamma astronomy, an instrument with a high sensitivity of $\sim 1$ mCrab $\left(3 \times 10^{-12} \mathrm{erg} \mathrm{cm}^{-2} \mathrm{~s}^{-1}\right.$ at $1 \mathrm{MeV}$ in $\left.10^{6} \mathrm{~s}\right)$ is desired.

Historically, COMPTEL operated with only $\sim 1 / 3$ of the sensitivity expected from the calibration test before launch, and it may give a concern in estimating the true sensitivity in orbit. The primary two causes of this discrepancy in COMPTEL are now understood as huge backgrounds in space and unclearness in the Compton images (Schönfelder et al. 2000; Schönfelder 2004).

After the close of the COMPTEL observations, it was pointed out that the additional parameters, including time of flight, kinematical tests, and Scatter Plane Deviation (SPD) from the direction of the recoil electrons, would be necessary to 


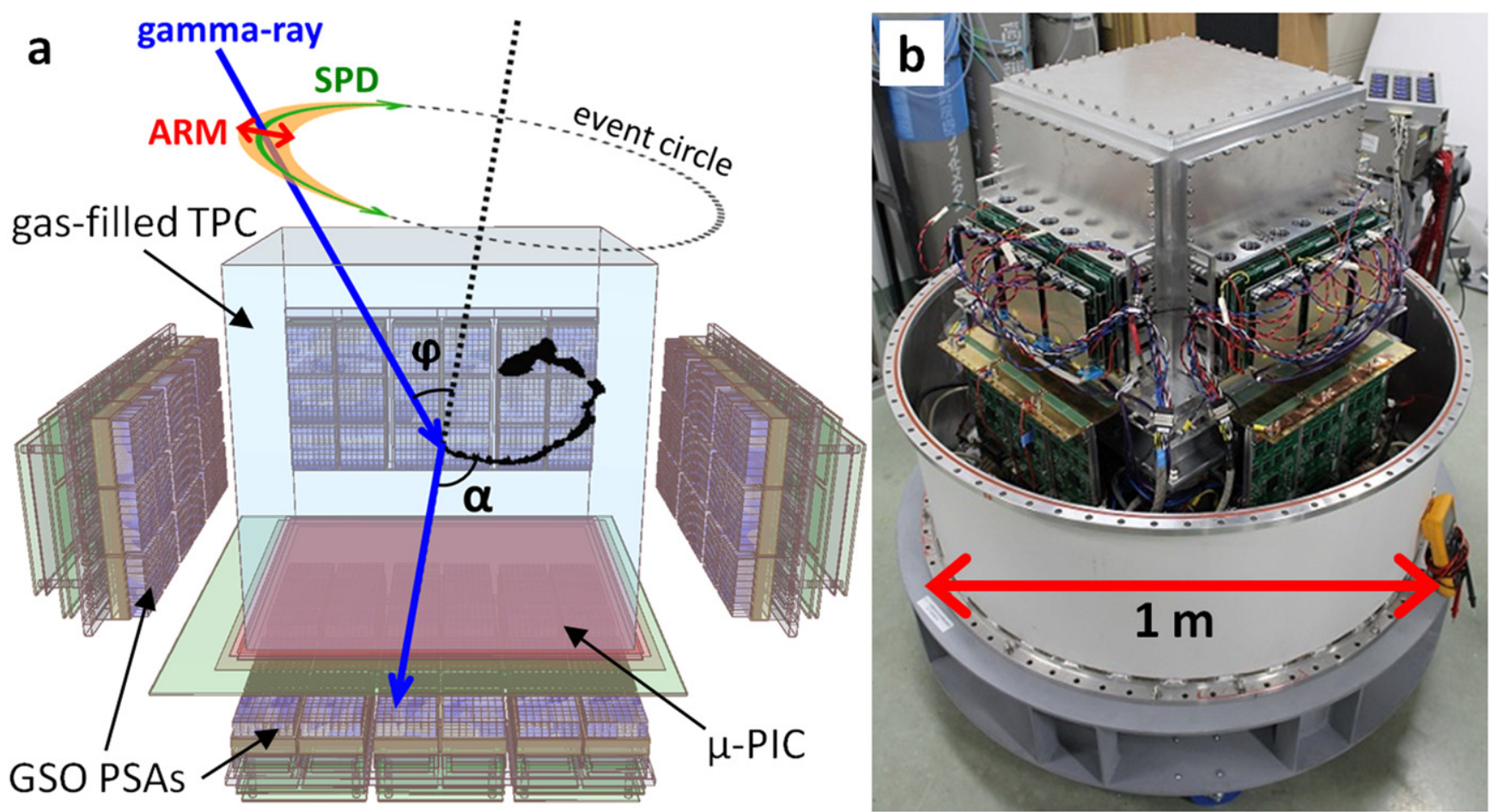

Figure 1. (a) Schematic view of SMILE-II $30 \mathrm{~cm}$-cubic ETCC. A micro-pattern gas detector (MPGD), which consists of $400 \mu \mathrm{m}$ pitch pixels, is installed at the bottom of the TPC, of which anodes and cathodes are connected via strips to provide the two-dimensional position and charge of the track. One PSA consists of 64 GSO bars (bar size: $6 \times 6 \times 13 \mathrm{~mm}^{3}$ ) with 1 radiation length (R.L.). 36 PSAs are put at the bottom and 18 PSAs are on each side. A typical reconstructed track of a recoil electron is plotted in Figure 1(a), using an improved reconstruction method, where the vertical width of the hit point represents the TOT as a pulse width of each pixel. (b) Photograph of SMILE-II flight model instrument.

reduce the background for the next-generation Compton cameras (CCs) (Schönfelder 2004). It is well known that in COMPTEL the time of flight between the forward and backward detectors dramatically reduced the background and was a primary factor for the success of COMPTEL. Ideally, the point-spread function (PSF) of CCs must be evaluated, based on the two parameters of angular resolution measure (ARM) and SPD, as shown in Figure 1 However, SPD is lost in CCs, due to lack of direction information of the recoil electrons, thus the PSF shape is inevitably assumed to be rather spread (see Section 6). The recovery of the SPD by measuring recoilelectron tracking is then expected to both (1) improve the measured PSF by up to several degrees and (2) reduce a substantial amount of contamination of the background leaked to the source region from the outside. In addition, the additional parameters of $d E / d x$ in recoil-electron tracking are independent of the reconstruction of Compton events, and therefore, use of them would suppress the background dramatically without losing Compton events.

In contrast, $\mathrm{CCs}$ with no additional parameters may lose a significant amount of Compton events in intense backgrounds because the application of cuts on physical parameters, such as energy deposits or hit positions, produces a massive uncertainty for the sensitivity. Nevertheless, the recent trend in developing advanced CCs concentrates on pursuing a larger effective area and a better energy resolution to improve the ARM resolution. In fact, Aprile et al. (2004) reported no detection of Crab in a balloon experiment with liquid $\mathrm{Xe} \mathrm{CCs}$ that has the largest effective area of $20 \mathrm{~cm}^{2}$ (Aprile et al. 2004). The NCT balloon experiment with the Ge-based CC and BGO shield detected Crab with $4 \sigma$ (Bandstra et al. 2011), although the reported number of signals from the Crab region was $\sim 1 / 6$ of simulation over a large background. Recent satellite proposals in $\mathrm{MeV}$ astronomy, in which the proposed sensitivities are larger than several mCrab, mention little on either of these problems (Boggs 2006; Greiner et al. 2009; Nakazawa et al. 2012; von Ballmoos et al. 2012). As such, it seems to be difficult for even advanced CCs to reach the desirable sensitivity in space.

To achieve the desired high sensitivity, combination of the sharp PSF and the reduction of the background with the SPD and additional parameters from recoil-electron tracking is a promising approach, given a $\mathrm{MeV}$ instrument with the effective area of several tens of $\mathrm{cm}^{2}$ will reach a sensitivity of $\sim 1 \mathrm{mCrab}$ if perfect background rejection is applied. Only a few studies on CCs with recoil electron tracking have been reported so far (Kanbach et al. 2006; Vetter et al. 2011). Among them, SMILE-I ("Sub-MeV gamma-ray Imaging Loaded-on-balloon Experiment": first Electron-Tracking Compton Camera; ETCC) showed the possibility to remove most of the background without an active shield by recoil electron tracking in a gaseous time projection chamber (TPC; Takada et al. 2011a).

Here we present the performance of an improved $30 \mathrm{~cm}$ cubic ETCC for our second balloon experiment (SMILE-II). We have successfully made robust reduction of background and obtained clear images by 3D electron tracking. In Section 2, the concept and structure of the ETCC are concisely explained. Then its fundamental performance is presented in Section 3. The durability of the ETCC with excellent ability of reducing background under intense radiation condition is mentioned in Section 4. In Section 5, an improvement of imaging, thanks to the use of SPD, is described in detail, and the definition of the PSF of the ETCC to reach the ultimate sensitivity of the CC is thoroughly discussed in Section 6. Finally, we summarize the 
Table 1

Specifications of the Instruments in Figure 3.

\begin{tabular}{|c|c|c|c|c|c|c|}
\hline Instrument & $\begin{array}{l}\text { TPC Size } \\
\left(\mathrm{cm}^{3}\right)\end{array}$ & $\begin{array}{c}\text { Gas Parameters } \\
\text { Mixture, Pressure, Drift Field }\end{array}$ & $\begin{array}{l}\text { Drift Velocity } \\
\left(\mathrm{cm} \mu \mathrm{s}^{-1}\right)\end{array}$ & $\begin{array}{c}\text { Diffusion }(\mu \mathrm{m} / \sqrt{\mathrm{cm}}) \\
\text { Transverse/Longitudinal }\end{array}$ & $\begin{array}{l}\text { Anode-cathode } \\
\text { Coincidence }\end{array}$ & PSAs \\
\hline SMILE-I type prototype & $10 \times 10 \times 8$ & $\begin{array}{l}\mathrm{Ar} 90 \% / \mathrm{C}_{2} \mathrm{H}_{6} 10 \%, \\
1 \mathrm{~atm}, 400 \mathrm{~V} \mathrm{~cm}^{-1}\end{array}$ & 4.0 & $470 / 230$ & $\begin{array}{c}\text { online } \\
10 \text { ns gate }\end{array}$ & $\begin{array}{l}\text { GSO } \\
1 \text { R.L. }\end{array}$ \\
\hline SMILE-I Flight Model & $10 \times 10 \times 15$ & $\begin{array}{c}\mathrm{Xe} 54 \% / \mathrm{Ar} 40 \% / \mathrm{C}_{2} \mathrm{H}_{6} 6 \% \\
1 \mathrm{~atm}, 380 \mathrm{~V} \mathrm{~cm}^{-1}\end{array}$ & 2.4 & $500 / 290$ & $\begin{array}{l}\text { online } \\
10 \mathrm{~ns} \text { gate }\end{array}$ & $\begin{array}{l}\text { GSO } \\
1 \text { R.L. }\end{array}$ \\
\hline SMILE-II small prototype & $7.5 \times 7.5 \times 15$ & $\begin{array}{l}\mathrm{Ar} 90 \% / \mathrm{C}_{2} \mathrm{H}_{6} 10 \% \\
1 \mathrm{~atm}, 170 \mathrm{~V} \mathrm{~cm}^{-1}\end{array}$ & 3.6 & $470 / 300$ & $\begin{array}{c}\text { offline } \\
\text { adequate gate }\end{array}$ & $\begin{array}{l}\text { GSO } \\
1 \text { R.L. }\end{array}$ \\
\hline SMILE-II (PSAs improved) & $30 \times 30 \times 30$ & $\begin{array}{c}\operatorname{Ar} 95 \% / \mathrm{CF}_{4} 3 \% / \text { iso- }_{4} \mathrm{H}_{10} 2 \% \\
1 \mathrm{~atm}, 160 \mathrm{~V} \mathrm{~cm}^{-1}\end{array}$ & 6.5 & $300 / 300$ & $\begin{array}{l}\text { offline } \\
\text { adequate gate }\end{array}$ & $\begin{array}{l}\text { GSO } \\
3 \text { R.L. }\end{array}$ \\
\hline SMILE-III & $30 \times 30 \times 30$ & $\begin{array}{c}\mathrm{CF}_{4} 100 \% \\
1 \mathrm{~atm}, 160 \mathrm{~V} \mathrm{~cm}^{-1}\end{array}$ & 5 & $100 / 100$ & $\begin{array}{c}\text { offline } \\
\text { adequate gate }\end{array}$ & $\begin{array}{l}\text { GSO } \\
3 \text { R.L. }\end{array}$ \\
\hline Satellite-ETCC (1 module) & $50 \times 50 \times 50$ & $\begin{array}{c}\mathrm{CF}_{4} 100 \% \\
1 \mathrm{~atm}, 160 \mathrm{~V} \mathrm{~cm}^{-1}\end{array}$ & 5 & $100 / 100$ & $\begin{array}{c}\text { offline } \\
\text { adequate gate }\end{array}$ & $\begin{array}{r}\mathrm{LaBr}_{3} \\
10 \text { R.L. }\end{array}$ \\
\hline
\end{tabular}

Note.

All of the drift velocities and diffusion constants were calculated using the MAGBOLTZ simulation code (Biagi 1999).

characteristics of the ETCC briefly and consider the prospects for the future MeV gamma-ray astronomy with the use of advanced ETCC.

\section{ELECTRON-TRACKING COMPTON CAMERA}

An ETCC consists of a TPC based on a micro pattern gas detector (MPGD) with $400 \mu \mathrm{m}$ pitch pixels for 3D tracking of recoil electrons and $\mathrm{Gd}_{2} \mathrm{SiO}_{5}: \mathrm{Ce}(\mathrm{GSO})$ pixel scintillator arrays (PSAs) to measure scattered gamma rays (Tanimori et al. 2004). With an ideal effective area of $100 \mathrm{~cm}^{2}$ at $1 \mathrm{MeV}$ for a $50 \mathrm{~cm}$-cubic $3 \mathrm{~atm} \mathrm{CF}_{4}$ gas, such a TPC would be a good device for a $\mathrm{CC}$ with electron-tracking. $\mathrm{CF}_{4}$ gas is an ideal gas for ETCC, with significant benefits, including a large cross section for Compton scattering, small photo-absorption cross section, and small diffusion constant (see Section 6 for detail). Several time-projection chambers have already been operated with pure $\mathrm{CF}_{4}$ in accelerators and underground experiments (Thun 1988; Isobe et al. 2003; Miuchi et al. 2010). However, we need more study to use the $\mathrm{CF}_{4}$-dominant gas in the MPGD-based TPC, due to the requirement of a higher-voltage electric field on the MPGD. In particular, good reliability for the stable operation of the TPC is crucial in the balloon experiments, and hence we at present use Ar-based gases with some additional quencher gases to study the effectiveness of electron tracking. Table 1 summarizes the detailed components of the Ar-based gases used in our experiments.

3D tracking of the recoil electron provides the incident gamma-ray direction as an arc by adding SPD to ARM, the additional angle of $\alpha$ in Figure 1(a), and the energy-loss rate $(d E / d x)$ of the track, all of which are effective tools to reduce backgrounds by directional cut, kinematical tests and particle identification, respectively. Note we have already examined the concept of ETCC (Orito et al. 2004; Tanimori et al. 2004; Takada et al. 2005), including an ARM resolution of $4^{\circ}$ (FWHM) at $662 \mathrm{keV}$ with $\mathrm{LaBr}_{3}$ PSAs for medical imaging (Kabuki et al. 2010; Kurosawa et al. 2010).

The readout method of the TPC is a key technology of this balloon experiment, as described in Tanimori et al. (2004) and Takada et al. (2011a). Only one or a few tracks, including background for Compton events, is found to appear in the TPC. Given that conventional orthogonal strip electrodes could be used, instead of pixel electrodes (as are used in typical TPCs), to reconstruct the $3 \mathrm{D}$ tracks by slicing along the direction of the electron drift using fast timing and combing the anode and cathode hits that have the same times (Tanimori et al. 2004), we could reduce the number of readout electrodes of the TCP by an order of two for this $30 \mathrm{~cm}$-cubic ETCC. This is an allowable level using recent ASIC and FPGA, while satisfying the severe constraint of balloon experiments for power consumption, space, and costs. However, such a drastic reduction of the number of signals would also introduce a risk of increasing the ambiguity of electron tracking, as will be mentioned later.

For MeV astronomy, SMILE-I with a $10 \mathrm{~cm}$-cubic ETCC was performed in 2006. We selected 400 downward gamma rays from $2.3 \times 10^{5}$ triggered events, using the kinematical test and $d E / d x$ (Takada et al. 2011a), where a kinematical test was made with comparison of the measured angle $\alpha$ to the calculated one, $\Delta_{\alpha}$, as mentioned in Section 2 of Takada et al. (2011a). Although both the resolution for $d E / d x$ and the detection efficiency were inefficient, due to a low tracking efficiency of $\sim 10 \%$, in which at least 5 hit points on each track were required, the combination of the $d E / d x$ cut and kinematical test was found to be useful for the reduction of neutrons and cosmic rays. This inefficiency was not due to the low gain of the MPGD but to a bug in the algorithms set in the FPGA used in the readout electronics (see the next section for details). The obtained diffuse atmospheric and cosmic fluxes were consistent with past observations. In addition, it is noted that its cubic structure provides a large field of view (FOV) of $2 \pi$ str.

\section{BASIC PERFORMANCE OF THE SMILE-II ETCC}

We have used a $30 \mathrm{~cm}$-cubic ETCC Figure 1(b) to establish a high sensitivity with a balloon experiment for the detection of the Crab (SMILE-II) (Takada et al. 2011b). To detect Crab with a reliable $\sim 5 \sigma$ significance in an exposure of several hours, an effective area of $0.5 \mathrm{~cm}^{2}$ at $300 \mathrm{keV}$ and an ARM resolution of 

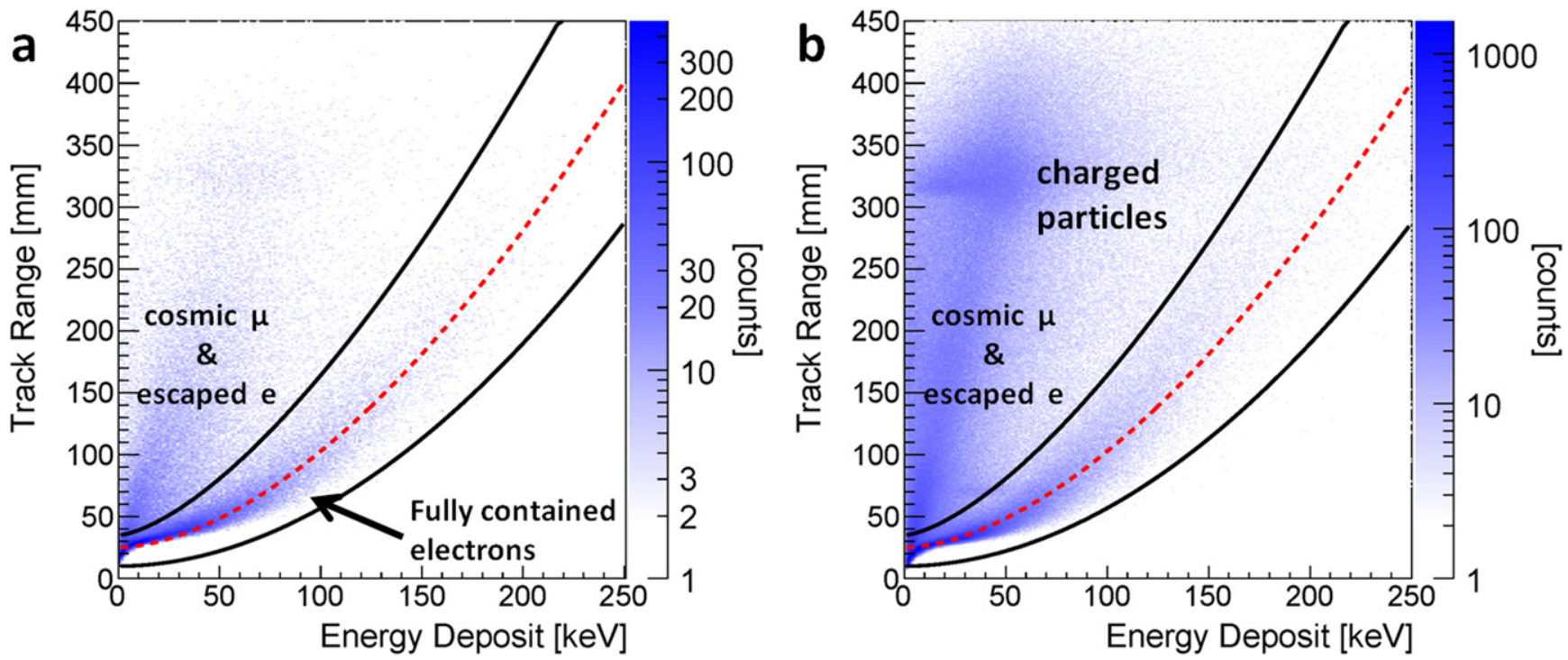

Figure 2. Correlations of the track length and energy deposit in the TPC (a) under the condition of gamma-ray irradiation from ${ }^{137} \mathrm{Cs}(3 \mathrm{MBq})$ at a distance of $1 \mathrm{~m}$ in the laboratory, (b) under the intense radiation generated by a $140 \mathrm{MeV}$ proton beam. In all figures, the fully contained electrons are clearly separated from the minimum ionizing charged particles, e.g., cosmic muons, high-energy electrons, and neutron-recoil events.

$<10^{\circ}(\mathrm{FWHM})$ at $662 \mathrm{keV}$ are required (Tanimori et al. 2012; Sawano et al. 2014).

The improvement of the reconstruction of tracks in the TPC was an essential factor for SMILE-II. Only addresses of the anode and cathode strips hitting simultaneously within a $10 \mathrm{~ns}$ gate were encoded for SMILE-I. However, a $10 \mathrm{~ns}$ gate is too short to get all hit strips, due to the delay in the encoding circuit. This was the reason for the small effective area of SMILE-I. To recover all hit points in SMILE-II, all addresses of the hit strips on anode and cathode with their hit timings were recorded without requiring the coincidence of $10 \mathrm{~ns}$, and an adequate gate is applied in the analysis (Tanimori et al. 2012). Additionally, a pulse height of each strip was recorded as a timing width over the threshold (TOT). Figure 1(a) shows a typical reconstructed track of a recoil electron for the TPC of SMILE-II, using an improved reconstruction method, where the vertical width of the hit point represents the TOT as a pulse width of each pixel. To measure the precise energy deposit in the TPC, a sum of 64 strips of both cathodes and anodes are fed to Flash ADC $(25 \mathrm{MHz})$, and its wave form was recorded during $10 \mu \mathrm{s}$. Also, two neighboring strips of both anodes and cathodes were combined to increase the pulse height. Then the track reconstruction efficiency was dramatically improved from $\sim 10 \%$ to $100 \%$, which provides a much better resolution for $d E / d x$, as demonstrated in Figure 2(a) (Mizumura et al. 2014), compared to that of SMILE-I (Figure 7 in Takada et al. 2011a). The details of the performance of this TPC is described in Matsuoka et al. (2015).

Figure 2(a) shows the track range and its energy deposit for $662 \mathrm{keV}$ gammas from ${ }^{137} \mathrm{Cs}$ source set at a distance of $1 \mathrm{~m}$ from the ETCC, in which $d E / d x$ is a gradient of the distribution. The measured $d E / d x$ in this figure clearly distinguishes the Compton electrons fully contained in the TPC from the backgrounds, and resultantly enables us to remove most of the backgrounds without loss of Compton events by applying cuts. The red-dashed line in this figure is the empirical formula of $d E / d x$, which is approximately proportional to $E^{1.72}$ (Sauli 1977), for recoil electrons fully contained in the TPC. The region within $E^{1.72 \pm 0.22}$ contains $\sim 95 \%$ of Compton events inside ( $d E / d x$ cut), as supported by the simulation result. In this article, all simulations were done using GEANT4 (Agostinelli et al. 2003). The simulation also shows the distribution of higher energy particles, such as, electrons escaping from the TPC and minimum ionizing particles (cosmic muons) on the upper boundary, as actually seen in Figure 2(a). In addition, the scattering point of the track is required to be within $1 \mathrm{~mm}$ inside the drift region of the TPC in order to remove events originated from gammas that scattered from the wall of the drift cage of the TPC (fiducial cut), with which $\sim 10 \%$ of the events passing the $d E / d x$ cut were removed. Thus, by applying only two simple cuts, almost all of the fully contained Compton events are obtained, after several kinds of backgrounds are filtered out. For the obtained fully contained Compton events, effective areas were measured at several energy points, using RI sources of ${ }^{139} \mathrm{Ce},{ }^{133} \mathrm{Ba},{ }^{22} \mathrm{Na},{ }^{137} \mathrm{Cs}$, and ${ }^{54} \mathrm{Mn}$, along with the simulation results for several types of ETCCs, where the effective area was obtained from the number of detected gammas within twice the FWHM of the energy resolution centered at the gamma energy in the background-subtracted energy spectrum. The simulation results were obtained mainly from the production of two probabilities: that of Compton scattering and its recoil electron contained fully in the TPC, and that of the full absorption of scattered gammas in the PSAs, where the absorption of the materials of the TPC and the supporting frames are taken into account.

Figure 3 shows good consistency between the measured efficiencies for the ETCCs listed in Table 1 (shown as points) and their simulation results (shown as lines). Here the $10 \mathrm{~cm}-$ cubic SMILE-II ETCC prototype (blue inverted-triangle points and yellow line), $30 \mathrm{~cm}$-cubic SMILE-II flight model ETCC (purple circles and red line), improved flight model with 3 radiation length (R.L.) GSO PSAs covering the bottom half of the TPC (green dot-dashed line), and SMILE-III ETCC (blue dashed line; described in the later section) are given. In addition, a $50 \mathrm{~cm}$-cubic ETCC with $\mathrm{CF}_{4}$ gas at 3 atm and 10 R.L. PSAs $(\Delta E / E \sim 4 \%$ at $662 \mathrm{keV}$ : FWHM) is plotted (black dotted line), in which the PSAs are set within the pressure vessel to catch high-energy electrons escaping the gas 




Figure 3. Energy dependencies of several measured and simulated effective areas drawn as points and lines, respectively. SMILE-I prototype (magenta squares), SMILE-II small $10 \mathrm{~cm}$-cubic ETCC prototype (blue inverted-triangle points and yellow line), SMILE-II $30 \mathrm{~cm}$-cubic ETCC flight model (purple circles and red line), improved one with 3 R.L. GSO PSAs (green dot-dashed line), a $30 \mathrm{~cm}$-cubic ETCC of SMILE-III (blue dashed line), and a $50 \mathrm{~cm}$-cubic ETCC (black dotted line). The effective areas were measured at several energy points, using RI sources of ${ }^{139} \mathrm{Ce},{ }^{133} \mathrm{Ba},{ }^{22} \mathrm{Na},{ }^{137} \mathrm{Cs}$, and ${ }^{54} \mathrm{Mn}$. The detailed configurations of these ETCCs are described in Table 1. Additionally, the yellow and blue stars are the effective areas of the Figure 6(b) beam experiment and Figure 7(b) Crab simulated measurement, respectively. The black square is explained in Figure 6(d).

volume of the TPC. A large FOV of $2 \pi$ str similar to SMILE-I was confirmed for the $30 \mathrm{~cm}$-cubic ETCC (Matsuoka et al. 2015). Thus, we are able to estimate precisely the effective area for the extensions of the volume, gas type and pressures of the TPC, or the R.L., and the better energy resolution of PSAs. These simple extensions ensure an effective area of $11 \mathrm{~cm}^{2}$ at $300 \mathrm{keV}$ for a following balloon experiment with a long duration flight (SMILE-III) and that of $60 \mathrm{~cm}^{2}$ with $2^{\circ}$ (ARM, FWHM) at $1 \mathrm{MeV}$ for a $50 \mathrm{~cm}$-cubic ETCC with $\mathrm{CF}_{4}$ gas at $3 \mathrm{~atm}$ and $\sim 10$ R.L. PSAs, e.g., $\mathrm{LaBr}_{3}$. Furthermore, a satelliteETCC consisting of four $50 \mathrm{~cm}$-cubic ETCCs for a middleclass satellite would reach $240 \mathrm{~cm}^{2}\left(4 \times 60 \mathrm{~cm}^{2}\right)$ with an ARM resolution of $2^{\circ}$ at $1 \mathrm{MeV}$.

The ARM resolutions were obtained at the same energy points Figure 4(a) with those calculated, based on the detector energy resolution. The measured ARMs were close to the theoretical limit of the resolution expected for the detector energy resolutions. Figure 4 also gives the ARM resolutions for future ETCCs with scintillators with better energy resolution. The discrepancies between the measured and calculated ARM resolutions are considered to arise from the $\sim 8 \mathrm{~mm}$ uncertainty in the track for the scattering point of the gamma, due to the worsened 3D reconstruction of the recoil electron, as mentioned below.

Finally, an SPD can be determined for all the events, and we obtained a resolution of SPD of $200^{\circ}$ (FWHM) Figure 4(b), which is about two times worse than the expected SPD resolution, due to multiple scattering in Ar gas. The SPD is determined with a linear fitting of the entire track. Even a SPD with such a poor resolution is useful to improve the image quality (see Section 5 for details). The deterioration of the SPD resolution is mainly due to the well-known ambiguity from multi-hits on the orthogonal 2D strip readout in the TPC. Usually, $n$-hits on anode and cathode strips in the same timing generate $n^{2}$ hit points Figures 5(a) and (b), and thus a part of the track running horizontally to the $\mu$-PIC is obtained as a square instead of a line. In this analysis, a timing resolution of nearly $10 \mathrm{~ns}$ from the clock of the FPGA $(100 \mathrm{MHz})$ was used. To address this issue, improvement of the ETCC is being carried out (Sections 5 and 6).

\section{PERFORMANCE UNDER INTENSE RADIATION BACKGROUND}

In order to investigate quantitatively the performance of the ETCC under intense radiation conditions similar to those found in space, we performed a test using a $140 \mathrm{MeV}$ proton beam incident on a water target to produce a diffuse background of fast neutrons and $\mathrm{MeV}$ gamma-rays. The $30 \mathrm{~cm}$-cubic ETCC was placed at a distance of $1.3 \mathrm{~m}$ from the target, and direct gammas from the water target were shielded with lead blocks, allowing us to uniformly irradiate the ETCC (Matsuoka et al. 2015). The neutron-gamma ratio and overall intensity can be adjusted by altering the beam intensity and size of the water target. Details of the radiation condition created by a proton beam on a water target and its simulation will be discussed elsewhere. For the present measurement, the ratio of neutrons to gammas was estimated to be $\sim 1: 1$ with both the simulation and the observed spectra of a neutron monitor located near the water target. This is similar to the composition of the radiation field observed with SMILE-I at the balloon altitude at the middle latitude of the northern hemisphere (Takada et al. 2011a).

During the 5 day test, the ETCC was operated stably at the counting rates up to $1 \mathrm{kHz}$, or more than 5 times higher than that expected under balloon conditions (Matsuoka et al. 2015). Figure 2(b) shows the distribution of the measured $d E / d x$ (similar to Figure 2(a)), where the recoil protons due to scattering of fast neutrons are located at the far right of the plot. Here, the Compton electrons are clearly separated from the intense backgrounds, allowing us to reduce the background contribution by one order of magnitude (see the energy spectra in Figure 6(a)), using $d E / d x$ and fiducial cuts. In this way, we successfully obtained a clear image of a ${ }^{137} \mathrm{Cs}$ source $(662 \mathrm{keV}$ gammas) set at a distance of $1 \mathrm{~m}$ from the ETCC Figure 6(b). The image of the source appears at the expected position with a significance of $7.9 \sigma$ (SPD resolution of $200^{\circ}$ ), after gammas with energies in the range $662 \pm 66 \mathrm{keV}$ are selected, where the significance was estimated by comparing the spectrum of the ${ }^{137} \mathrm{Cs}$ source with the ring-shaped background region between $60^{\circ}$ and $90^{\circ}$ from the center of the FOV (i.e., the center of the target). It is also noted that the detection efficiency was consistent with that measured in the laboratory using the same cuts, as in Figure 3.

For comparison, we performed the image reconstruction using a conventional Compton imaging technique, including no information about the electron track Figure 6(c). In this case, only a small enhancement of $2.0 \sigma$ was obtained for the source signal. The significance could be increased to $5.4 \sigma$ by applying a tight cut on the electron energy measured in the TPC Figure 6(d). However, such a tight cut would also remove about $85 \%$ of Compton events, as compared to our analysis using the detailed information of the electron tracks, which corresponds to a decrease in the efficiency to $\sim 15 \%$ of the nominal value (as indicated with the black square in Figure 3).

This clearly demonstrates the effectiveness of the ETCC and the additional detailed tracking information for gamma-ray 

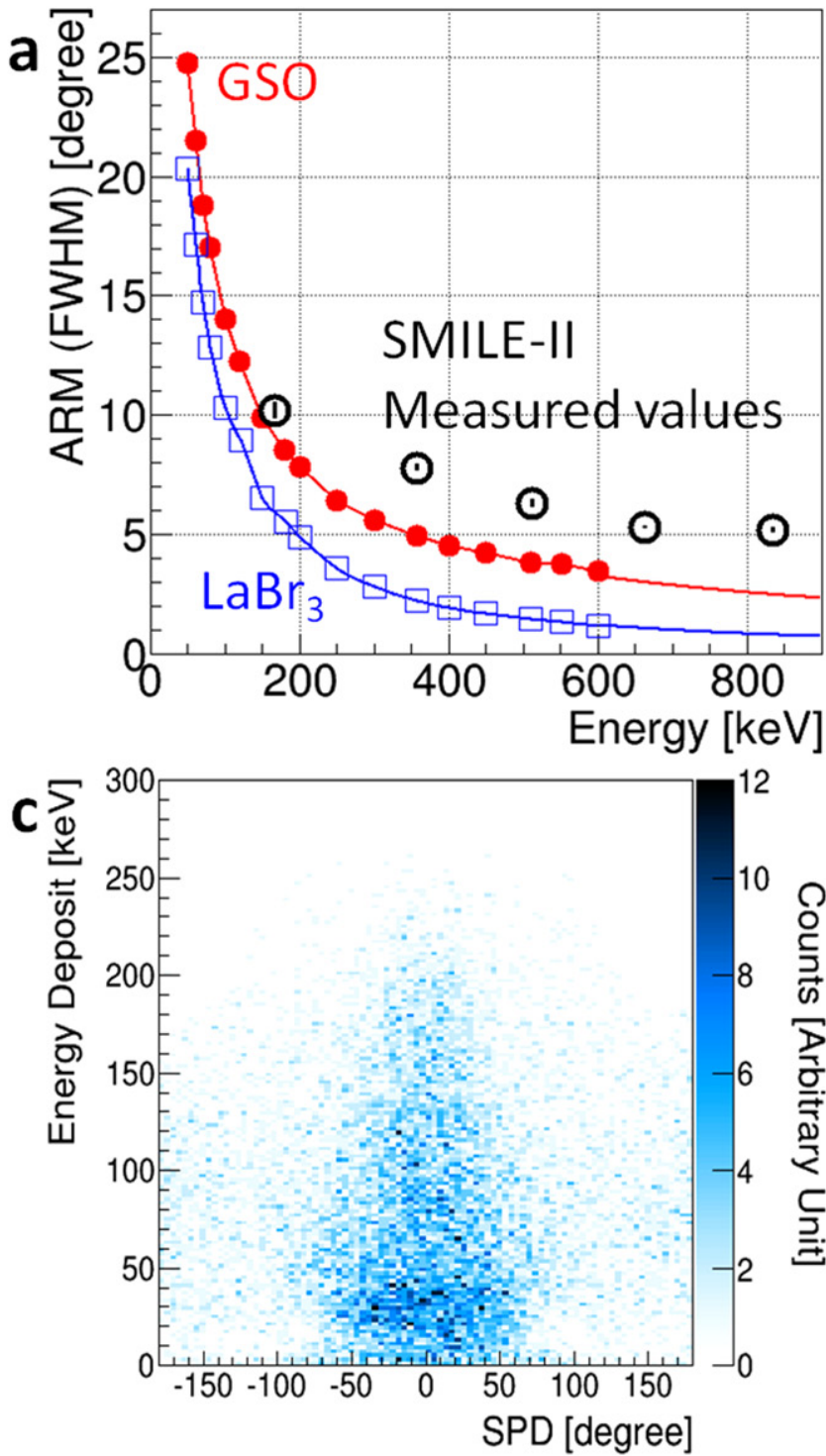

b

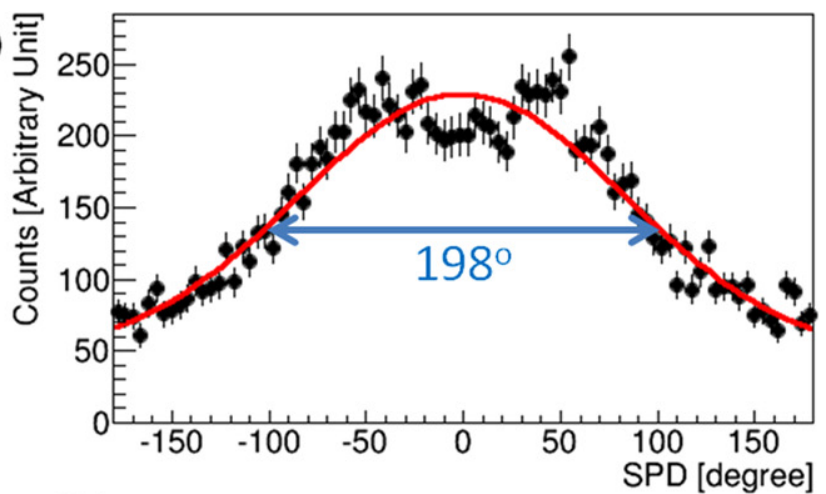

d

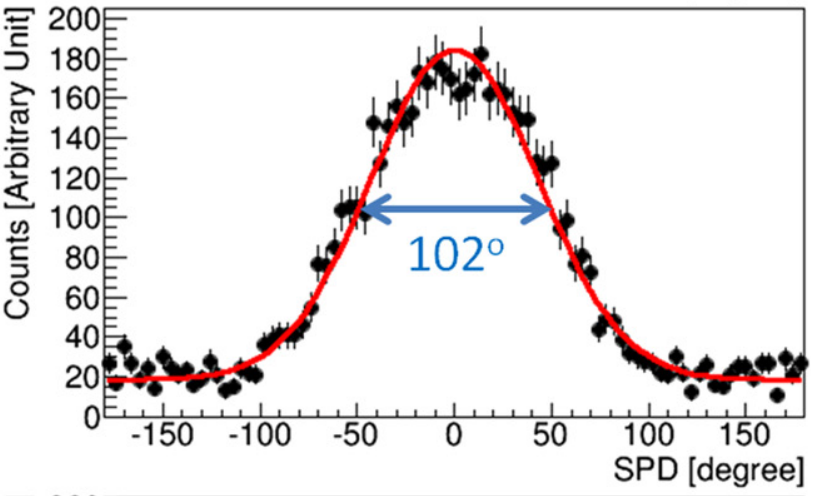

e

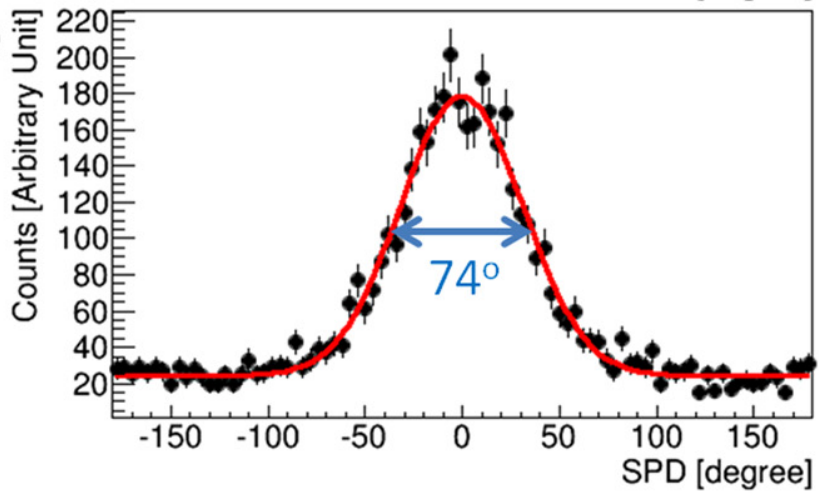

Figure 4. (a) Variation of the measured ARM resolutions and those simulated for GSO and $\mathrm{LaBr}_{3}$ PSAs are plotted with open circles, filled circles and open boxes, respectively. Here statistical errors of the measured ARM resolution are indicated within the plots, and simulated ARM resolutions are calculated from the energy resolutions of the TPC and PSAs. (b) The distribution of the SPD using the previous tracking method. (c) Correlation of the energy of a recoil electron and the SPD resolution after the improvement of the tracking method, and its projections on the SPD for (d) low energy recoil electrons (10-80 keV) and (e) high energy ones $(>80 \mathrm{keV})$. All the figures include some amount of background from gammas scattered between the ${ }^{137} \mathrm{Cs}$ source and the ETCC due to the worse energy resolution of GSO PSAs, which broaden the SPD resolution, compared to that due to directly incoming gammas.

imaging, as compared to traditional CC. The requirement of one fully contained electron in the TPC removes the major background sources of electron escape and reabsorption events. In addition, the $d E / d x$ cut effectively rejects all "neutrons and cosmic rays." Thus, we are able to select pure Comptonscattering events without loss of efficiency by applying these two filters, i.e., the fully contained recoil electrons and the $d E / d x$ cut (Figure 2). Such a robust event selection also gives the added benefit of enabling us to simulate the fluxes and images of gammas from both celestial points and extended objects with high reliability.

In a separate experiment, a measurement was carried out, using a $27 \mathrm{kBq}$ equivalent ${ }^{22} \mathrm{Na}$ source $(511 \mathrm{keV})$ set at a distance of $5.5 \mathrm{~m}$ from the ETCC, in order to study the performance under poor signal-to-noise ratio $(\mathrm{S} / \mathrm{N})$ conditions similar to those present in astronomical observations. This setup was expected to provide a $\mathrm{S} / \mathrm{N}$ of about a half of that expected from the observation of Crab under balloon conditions (expected $\mathrm{S} / \mathrm{N}$ of $\sim 0.005$ ). By applying the two cuts explained in Section 3, we obtained a clear image with a significance of $7.1 \sigma$ in $100 \mathrm{hr}$ observation for an SPD resolution of $200^{\circ}$ and gamma energies of $511 \pm 51 \mathrm{keV}$ Figure 7(b). Figure 7(a) gives the associated energy spectra. The error of the measured fluxes was $\sim 20 \%$, as estimated from the difference between the measurement result and that expected from the effective area in Figure 3. On the other hand, no significant enhancement $(<3 \sigma)$ was observed in the absence of electron-tracking information, even after a tight energy-cut is applied Figure 7(c). This result of Figure 7(b) supports a significant detection of Crab with at least $5 \sigma$ in the energy range of 200-600 keV in $4 \mathrm{hr}$ of observation, even with a poor SPD resolution of $200^{\circ}$, where we take into account by respective factors of 4 and 3 increases in the effective area and in the flux of Crab in the energy range of $200-400 \mathrm{keV}$. 

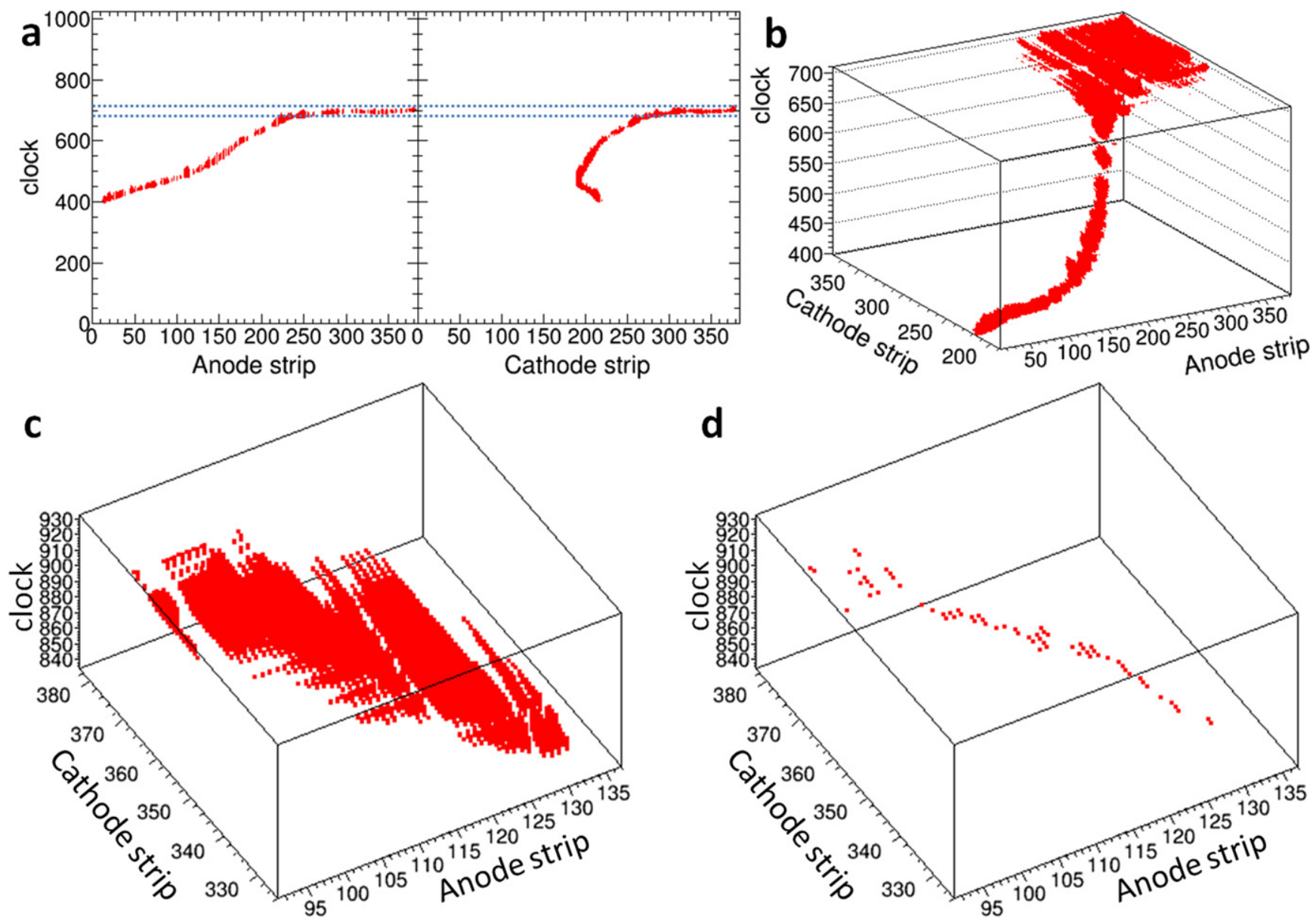

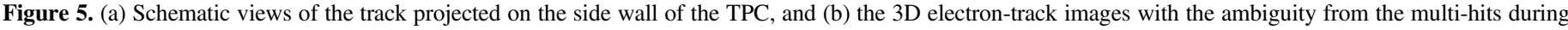
$10 \mathrm{~ns}$ indicated between two dotted blue lines on the orthogonal 2D strip readout, and typical electron track (c) before and (d) after the correction of time walk.

\section{IMAGING WITH USE OF SPD}

Unclean images are another serious problem in the CC. However it has been dramatically improved by determining the SPD for all the events.

In CCs, images are obtained by accumulating Compton annuli on the celestial sphere, where a fair amount of background event annuli overlap the target region and produce serious artifacts. Figures 8(a) and (b) show the significance maps of three ${ }^{137} \mathrm{Cs}$ sources with different intensities, put at a distance of $2 \mathrm{~m}$, with respective annuli and SPD arcs being overlaid. The significances are improved by a factor of $\sim 3$, even with the worst possible SPD resolution of $\sim 200^{\circ}$. To obtain these images, the probability functions of one gamma were defined as an annulus with the area normalized to 1 for a CC (Schönfelder et al. 1993) and as a Gaussian normalized with the SPD resolution for the ETCC.

The SPD resolution was determined by the uncertainty of the reconstruction method rather than the multiple scattering of a recoil electron (Section 2). We have recently successfully improved the time resolution of the coincidence between anodes and cathodes to $1 \mathrm{~ns}$ from $10 \mathrm{~ns}$ by correcting the time walk of each hit pixel using the TOT. As a result, the reduction of the number of multi hits in a track provides a very clear image of the track Figures 5(c) and (d). Such a clear track enables us to measure the SPD at a distance of $1 \mathrm{~cm}$ from the scattering point, which reduces the effect of multiple scattering in the gas. Figure 4(c) shows a correlation between the improved SPD and the electron energy. The spread of the SPD obviously shrinks, as the electron energy increases $(>80 \mathrm{keV})$ Figures 4(d) and (e). The SPD resolutions are nearly consistent with the multiple scattering angles in Ar gas. Next, we reanalyzed the case presented in Figure $8(\mathrm{~b})$ with a SPD resolution of $100^{\circ}$ and two SPD resolutions optimized for low energy electrons $(<80 \mathrm{keV})$ and high energy ones $(>80 \mathrm{keV})$, and plotted the results in Figures 8(c) and (d), respectively. We found a massive increase in the signal by $\sim 10 \sigma$. In Figure 8(d), the most reliable SPD resolution that maximizes the imaging significance was estimated for each energy range $(10-80 \mathrm{keV}$ and $>80 \mathrm{keV}$ ), although we should note that the significances are moderately dependent on the SPD resolutions. These values are better than the SPD resolutions in Figures 4(d) and (e) (see the caption of Figure 4).

\section{DISCUSSION}

Our ETCC provides a robust recipe for both removing the huge backgrounds and producing clear images, and as a result, gives nearly 10 times better overall sensitivity than that of conventional CC. The next important step would be the improvement of the SPD resolution up to the multiple scattering-angle in order to exploit the potential of electron tracking.

It is well known that the annulus of conventional CC leads to multiple intersections and a wide spread of the PSF. A better SPD resolution is naively expected to increase the significance 

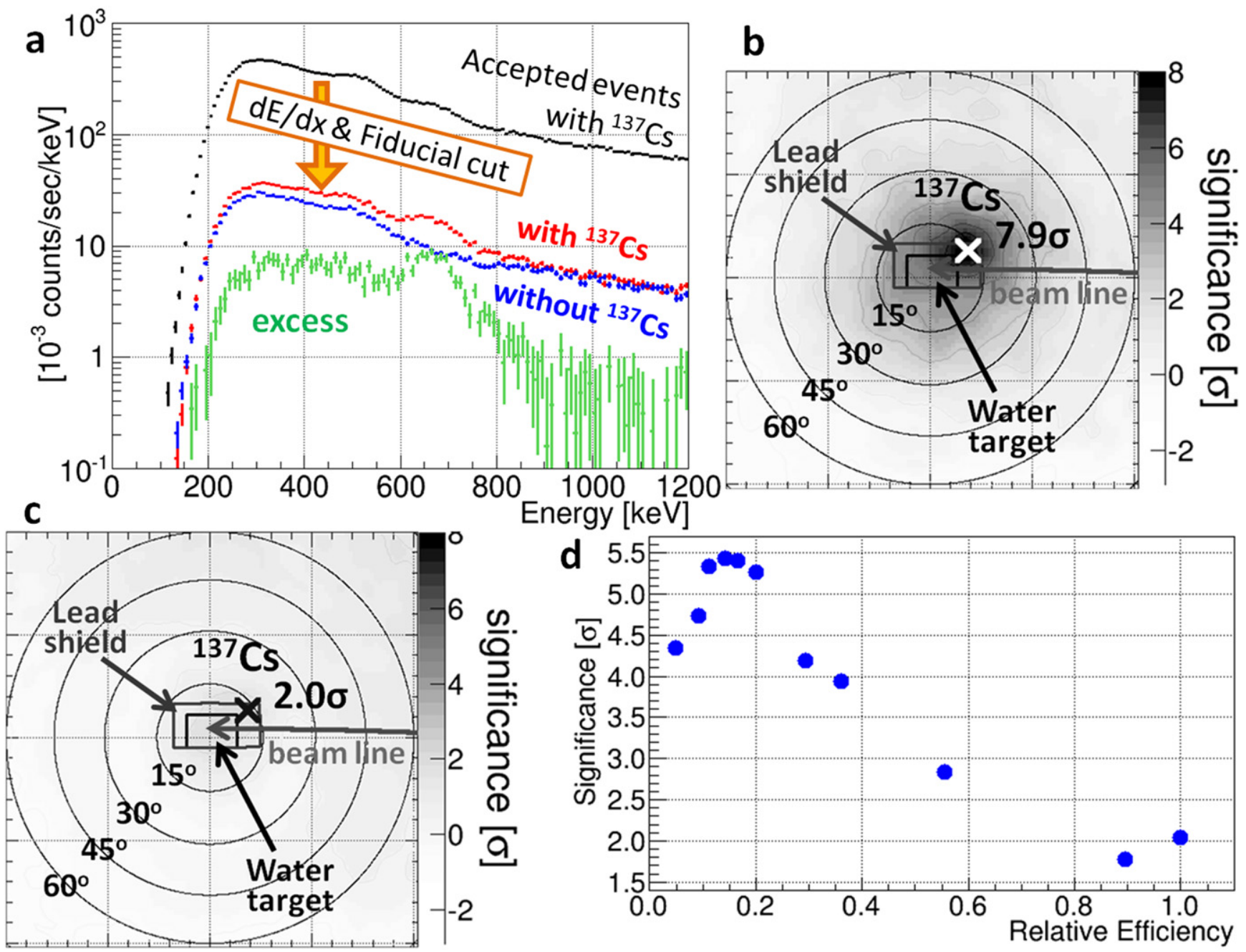

Figure 6. (a) Four energy spectra for reconstructed events under the intense radiation, which is generated by a $140 \mathrm{MeV}$ proton beam, before and after applying $d E / d x$ and fiducial cuts with a ${ }^{137} \mathrm{Cs}$ source, without ${ }^{137} \mathrm{Cs}$ as a background, and the spectrum of the excess gammas from ${ }^{137} \mathrm{Cs}$, which is derived by subtracting the background spectrum. In addition, (b) is the observed image of ${ }^{137} \mathrm{Cs}(0.8 \mathrm{MBq})$ set at a $1 \mathrm{~m}$ distance from the ETCC using electron tracking. (c) is a conventional Compton image without electron track information with the same data of (b). (d) Variation of the significance without electron track information as a function of the limiting ratio of the energy range of an electron in the TPC, where we apply additional cuts on the energy deposit in the TPC, and the horizontal axis is the ratio of the remained events after this cut to the events with the use of electron track information is applied.
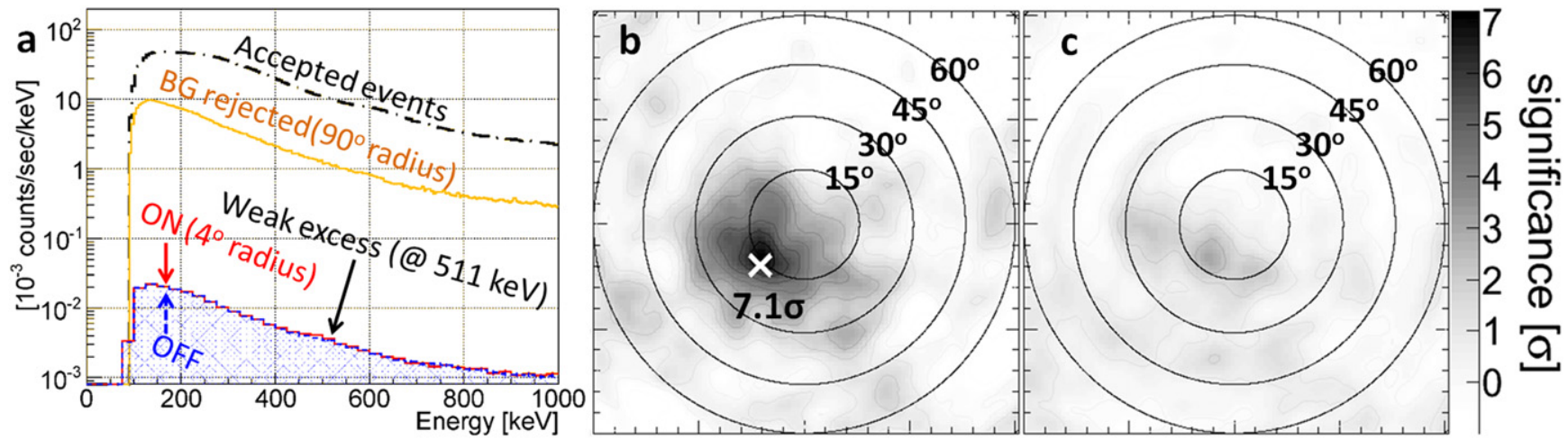

Figure 7. (a) Energy spectra of a super-weak point source $\left(27 \mathrm{kBq}{ }^{22} \mathrm{Na}\right)$ at a distance of $5.5 \mathrm{~m}$. (b) Observed image with $7.1 \sigma$ using electron tracking and SPD resolution of $200^{\circ}$ before the improvement, and in (c), no significant enhancement $(<3 \sigma)$ appears in the absence of electron tracking.

by a factor roughly proportional to $180^{\circ} /(\mathrm{SPD}$ resolution). Our simulation results appear to confirm it Figures 9(a) and (b), where a $\mathrm{S} / \mathrm{N}$ of $1: 10^{3}$ (or $10^{3}: 10^{6}$ total events) is assumed. In the simulation, the variation of the significance as a function of the SPD resolution was calculated for three accumulated regions on the FOV and two ARM resolutions, while constraining the Compton scattering angle $\varphi$ Figure 1(a) to smaller than $60^{\circ}$.

Figure 10 shows the calculated dependence of the multiple scattering angle of the recoil electron along the length of the 


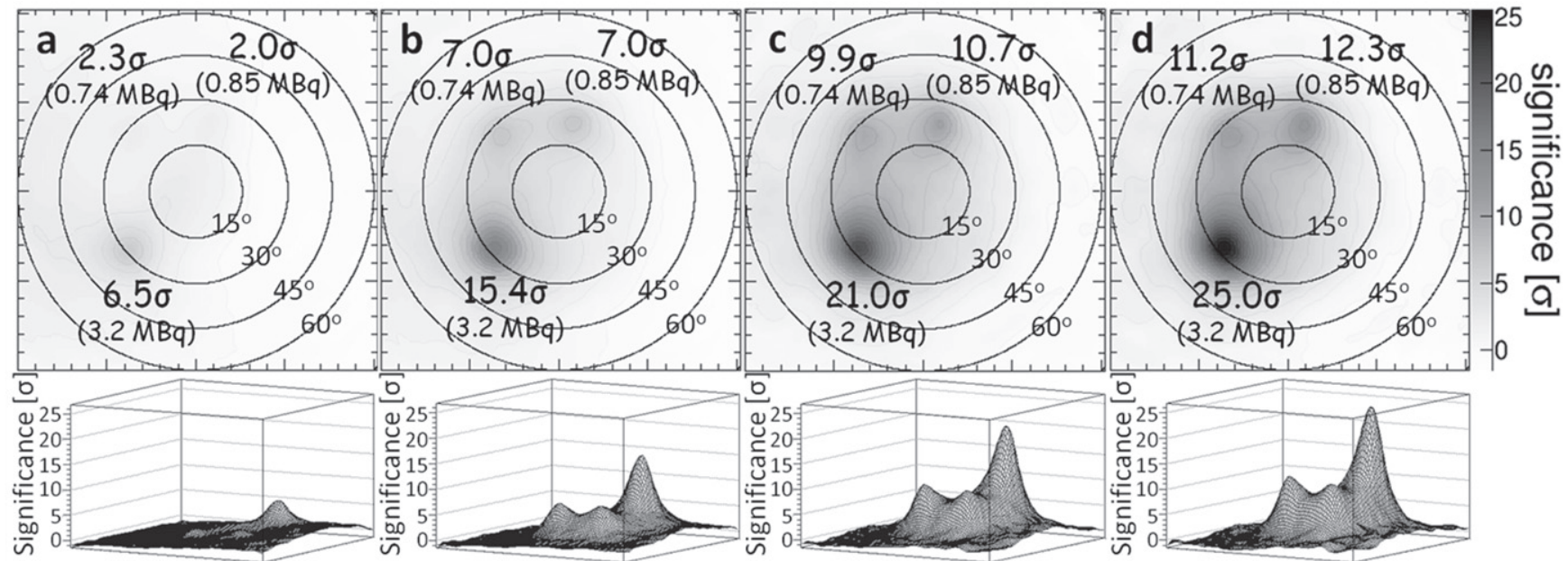

Figure 8. Significance maps and contours of three ${ }^{137} \mathrm{Cs}$ sources with different intensities at a distance of $2 \mathrm{~m}$, obtained by (a) accumulating the annulus without tracking information, and (b) using the arcs with SPD resolution of $200^{\circ}$ before the improvement. Same figures after the improvement are (c) and (d) for an SPD resolution of $100^{\circ}$ and the combination of SPD resolutions of $90^{\circ}$ (recoil electron energy range of $10-80 \mathrm{keV}$ ) and $45^{\circ}$ ( $>80 \mathrm{keV}$ ), respectively. For (d), the most reliable SPD resolution that maximizes the imaging significance was estimated for each energy range $(10-80 \mathrm{keV}$ and $>80 \mathrm{keV})$, although we should note that the significances are moderately dependent on the SPD resolutions. These estimated SPD resolutions were better than that in Figures 4(d) and (e), as mentioned in the caption of Figure 4.
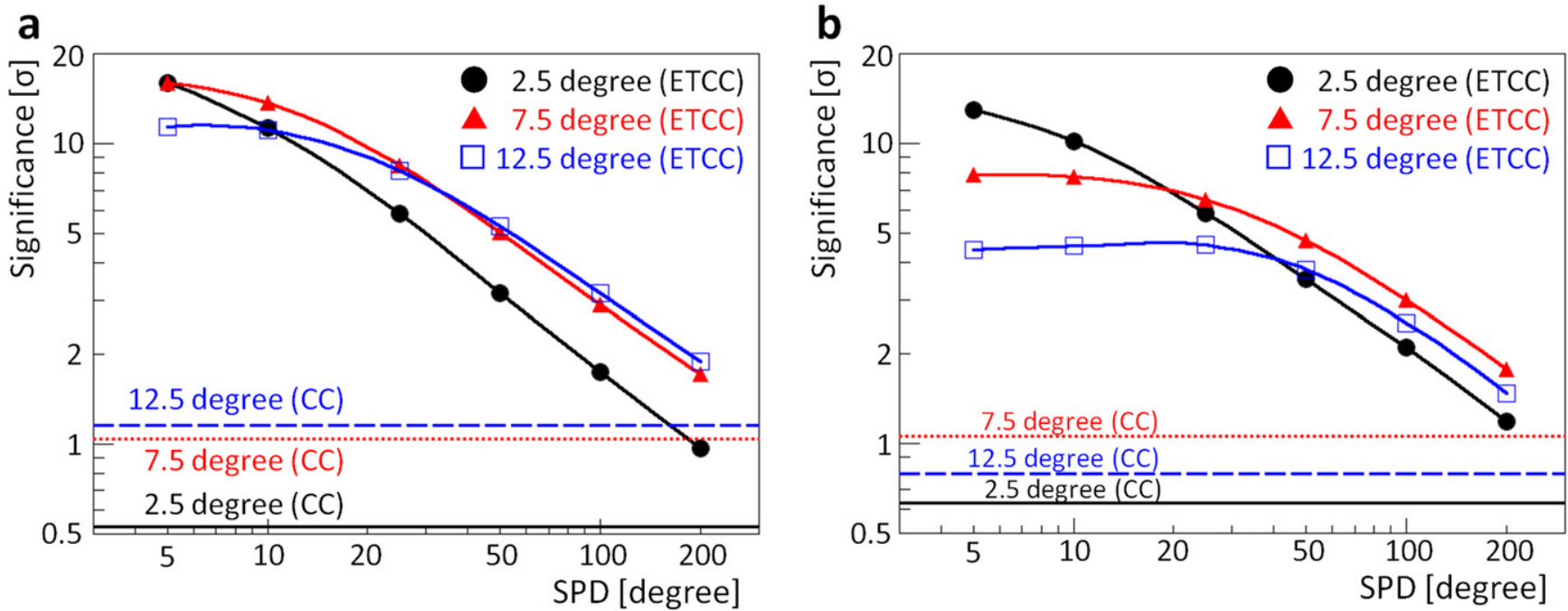

Figure 9. Simulated significances as a function of the SPD resolution (FWHM) for the signal to noise ratio of $10^{-3}$, using $10^{3}$ signal events and $10^{6}$ background events randomly distributed on the $2 \pi$ str, where three accumulated regions on the FOV are indicated with angular radii of 2.5 (circle), 7.5 (triangle) and 12.5 (box). The ARM resolutions of $2^{\circ}$ and $5^{\circ}$ (FWHM) are assumed for (a) and (b) respectively, and the Compton scattering angle $\varphi$ in Figure 1 (a) is restricted within $60^{\circ}$.

track used for the angle measurement for $\mathrm{Ar}$ and $\mathrm{CF}_{4}$ gases at the normal pressure and $\mathrm{CF}_{4}$ at $3 \mathrm{~atm}$, where the calculation was performed with GEANT4, in which we employed an empirical expression for the multiple scattering based on experimental data (Attwood et al. 2006). We expect to improve the SPD resolution to the hatched region in this figure (see the discussion later in this section). Thus, the SPD resolutions for gammas above $500 \mathrm{keV}$ (with recoil-electron energy of $>200 \mathrm{keV}$ ) and above $1 \mathrm{MeV}$ will be reduced to within $20^{\circ}$ and $10^{\circ}$, respectively.

The cumulative ratio of the PSF for gammas emitted from a point source is plotted as a function of its angular radius for three SPD resolutions $\left(100^{\circ}, 25^{\circ}\right.$, and $\left.5^{\circ}\right)$ in Figure 11 , along with the annulus of a conventional $\mathrm{CC}$, where two ARM resolutions of $2^{\circ}$ and $5^{\circ}$ (FWHM) are assumed. Hereafter, PSF $(\theta)$ is defined to contain a half of the gammas emitted from the point source within the angular radius $\theta$. First, from Figure 11, we note that the PSF is predominantly dependent on the worse one between the SPD and ARM resolutions, where an SPD resolution of $\sim 20^{\circ}$ gives a similar dispersion as an ARM resolution of $5^{\circ}$ when projected on the celestial sphere. The improvement in the significance due to better ARM resolutions is only seen for the case with the SPD resolution of better than $\sim 30^{\circ}$ (Figure 11). Thus, the improvement of the ARM resolution from $5^{\circ}$ to $2^{\circ}$ is effective in improving the PSF only if the SPD resolution is also improved below $\sim 30^{\circ}$. The PSF for conventional CCs, on the other hand, appears to be determined by the Compton scattering angle $\varphi$ of Figure 1(a), judging from the fact that the cumulative ratio of the PSF reaches 1 at $\sim 120^{\circ}$, or the largest diameter of the scattering $\varphi$. Thus, only an improved SPD resolution can counteract the spread of signals due to the annulus in a $\mathrm{CC}$, and can recover a point-like PSF similar to telescopes in other energy bands. 


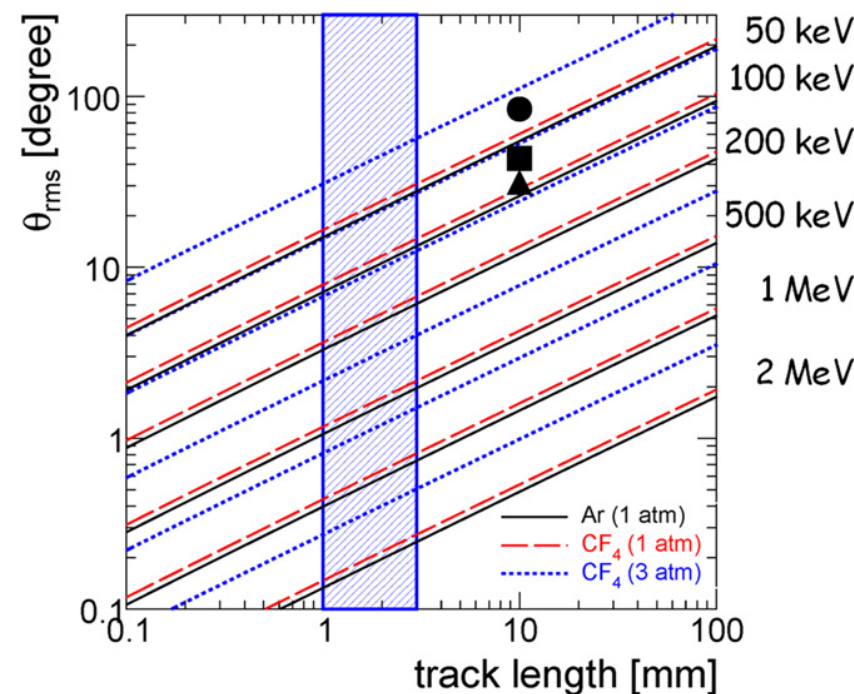

Figure 10. Dependence of the multiple-scattering angles of a recoil electron along the length of the track from the scattering point in $\mathrm{Ar}$ and $\mathrm{CF}_{4}$ gases with varying electron energies, where the normal pressure is assumed for both gases. The circle, square and triangle are the root mean square of SPD resolutions of $200^{\circ}$ (before improvement), $90^{\circ}$ and $45^{\circ}$ from Figure 8(d), respectively. The hatched region corresponds to the SPD resolutions expected by fine track sampling of $400 \mu \mathrm{m}$ pitch with a fine fitting.



Figure 11. Cumulative ratio in the PSF for gammas from a point source as a function of its angular radius for several SPD resolution and two ARMS; pastel red and black lines are the ARM resolutions of $2^{\circ}$ and $5^{\circ}$, respectively, and solid, dotted, dashed and dot-dashed lines are conventional CC, SPD resolutions of $5^{\circ}, 25^{\circ}$, and $100^{\circ}$, respectively.

The wide spread of the PSF for poor SPD resolutions and conventional CCs is due to the normalization of the probability on the arc and annulus (Section 5), which is necessary to keep the background level statistically similar for any SPD resolution, including an annulus. Maximum Likelihood Expectation Maximization (ML-EM) has been used in CCs to compensate for the spread and sharpen the PSF of gamma-ray sources from the background (Schönfelder et al. 1993; Wilderman et al. 1998; Bandstra et al. 2011). When the statistics of the signals are ideal, ML-EM can extract the maximum signal as determined by the effective area and PSF (ARM resolution). However, even with ML-EM, it is difficult to reproduce statistically poor signals from a huge background with the uncertainty at the similar level as the signal. We also note that an inherent risk of introducing artifacts for such backgrounddominated data should not be underestimated, particularly in $\mathrm{MeV}$ gamma-ray astronomy. Thus, conventional $\mathrm{CCs}$ have looked to detect signals for bright celestial objects only, for which the ML-EM works optimally. However, moderateintensity objects, whose flux was judged to be sufficient to allow detection, based on the sensitivity estimated from the effective area and PSF, actually turned out to be difficult to detect due to the breakdown of the ML-EM method. On the other hand, ML-EM would function well for an ETCC, thanks to its sharp PSF and low background. More fundamentally, the sensitivity would be determined solely by the effective area and the PSF, and supplemental tools, such as ML-EM, only serve to improve the significance by several factors by applying some constraints.

Considering all that, how would a better SPD resolution be attained, using the present technology of the ETCC? In the present detector technology, a gaseous TPC with a micropattern detector provides very accurate 3D tracking for electrons in the energy range from $\mathrm{keV}$ to $\mathrm{MeV}$. Even a high-resolution CCD with a $10 \mu \mathrm{m}$ pixel pitch and 2D tracking has been reported to provide an SPD resolution of only $\sim 200^{\circ}$ FWHM for $\sim 100 \mathrm{keV}$ for $2 \mathrm{D}$ tracking (Vetter et al. 2011). We have just begun the detailed study of the $3 \mathrm{D}$ reconstruction of recoil electrons in the gas of the ETCC, and expect to be able to improve the uncertainty of the Compton-scattering point from the current $\sim 8 \mathrm{~mm}$ down to a few millimeters. This will improve both the SPD and ARM resolutions to about a half of the present value and to the calculated value in Figure 5, respectively.

The selection of the gas for the TPC is the most important factor for the performance of the ETCC. In general, a scatterer of CCs should provide a large cross-section of Compton scattering, while also having a small cross-section for photo absorption to minimize the background. In addition, a small diffusion constant and small multiple scattering for the recoil electron are very important for the ETCC. All of the requirements above can be met with $\mathrm{CF}_{4}$ gas in the ETCC. Then, we are planning to use a $3 \mathrm{~atm} \mathrm{CF}_{4}$ gas in the next version of the ETCC. $\mathrm{CF}_{4}$ gas has a large Compton-scattering cross-section due to the large number of electrons per molecule (44), while simultaneously suppressing the photo-absorption cross-section, which is proportional to $Z^{5}$ in the Born approximation (with the atomic number $Z=9$ for $F$ ). Its diffusion constant is smaller than that of Ar gas. Hence, it is possible to develop a larger TPC with a drift length greater than $50 \mathrm{~cm}$, where a positional error at the MPGD due to the fluctuation of the centroid of the diffusive drift-electron cloud over a $50 \mathrm{~cm}$ drift length is estimated to be $\sim 150 \mu \mathrm{m}$ in a $3 \mathrm{~atm}$ $\mathrm{CF}_{4}$ gas.

Furthermore, with the use of $\mathrm{CF}_{4}$, the SPD resolution, which is the most important parameter for the ETCC, is expected to be improved in the following reason. For pressurized gas, the multiple scattering angle is proportional to the product of $\sqrt{ }$ (pressure) and $\sqrt{ }$ (track range). Whereas the multiple scattering angle per length of $\mathrm{CF}_{4}$ is similar to that of $\mathrm{Ar}$ gas (Figure 10), $d E / d x$ is 3 times larger, and the diffusion constant 
is smaller, than those of Ar gas. Then, combined with a smaller track sampling of $400 \mu \mathrm{m}$ (reduced from the present $800 \mu \mathrm{m}$ ), it would significantly improve the fitting resolution of the track. With this expected improvement, it would be possible to reduce the track range needed to measure the SPD from $1 \mathrm{~cm}$ down to a few $\mathrm{mm}$, which would exceed the increase of the multiple scattering due to the increase in gas pressure to $3 \mathrm{~atm}$. Thus, an ETCC with pressured $\mathrm{CF}_{4}$ gas would give a slightly better PSF than that of Ar gas at the normal pressure. This fact, combined with the properties described above, makes $\mathrm{CF}_{4}$ gas extremely well suited to provide the optimum performance for our ETCC.

Next, although the introduction of $d E / d x$ and SPD successfully resolved the two problems of huge backgrounds and unclear images, we should also consider the backgrounds that cannot be removed in theory with $d E / d x$ and/or SPD, i.e., the simultaneous emission of $\mathrm{X}$ - and gamma-rays or two gammarays from radioisotopes generated by cosmic rays, and accidental coincidences between the TPC and PSAs. Due to the large cross-section for photo absorption of X-rays, false Compton events arise from a photo-electric absorption of one gamma ray (or X-ray) in the TPC and a random coincidence of a second gamma ray in the PSA. Indeed, those false events were reported to be one of the dominant backgrounds in COMPTEL (Weidenspointner et al. 2001). However, the small photo-electric absorption cross-section of $\mathrm{CF}_{4}(\sim 1 / 5$ of that of $\mathrm{Ar}$ with the same number density of atoms) makes it considerably less sensitive to X-rays compared to $\mathrm{Ar}$ and $\mathrm{Si}$. Absorption probabilities for X-rays at an energy of a few tens of $\mathrm{keV}$ in a $50 \mathrm{~cm}$-cubic $3 \mathrm{~atm} \mathrm{CF}_{4}$ and $8 \mathrm{~atm}$ Ar are transparent $(\sim 10 \%$ at $30 \mathrm{keV})$ and opaque ( $>80 \%)$, respectively, where the respective pressures are chosen so that they have the same Compton-scattering probability. Such insensitivity to X-rays of $\mathrm{CF}_{4}$ also reduces the false Compton events, because most of the single hits in the TPC are due to X-rays. Furthermore, the ETCC provides another tool to remove such backgrounds via a kinematical test using the angle $\alpha$ of Figure 1(a), which was introduced as a new parameter for the ETCC in SMILE-I (Section 2). The poor angular resolution for the direction of the recoil electron would result in the rejection of a number of good Compton events. For that reason, we have not used the kinematic test for SMILE-II. However, because such events do not satisfy Compton kinematics, the angle $\alpha$ should be randomly distributed between $-90^{\circ}$ and $90^{\circ}$. Further improvement of the directional resolution of the recoil electron will reduce the uncertainty of $\alpha$ to $<20^{\circ}$ (from the current $\sim 40^{\circ}$ at FWHM), which will allow us to suppress such background by nearly one order of magnitude.

A final remark is that we have developed the ETCC, using well-established technologies (i.e., gas counters and scintillators), and even a 4-module satellite ETCC would need only $\sim 10^{4}$ readout channels for TPC and PSAs with low power electronics and no specific cooling system. In addition, due to the strong background rejection capability, no active shield would be required. Then, the component with the largest contribution to the weight would be the scintillators (more than half the total weight), which is intrinsically needed to absorb and detect gamma rays. Thus, the ETCC would be the lightest CC, and would provide the lowest background-radiation level in space.

\section{CONCLUSION}

We have revealed that the SPD dramatically recovers the gammas detected in the effective area within several degrees of

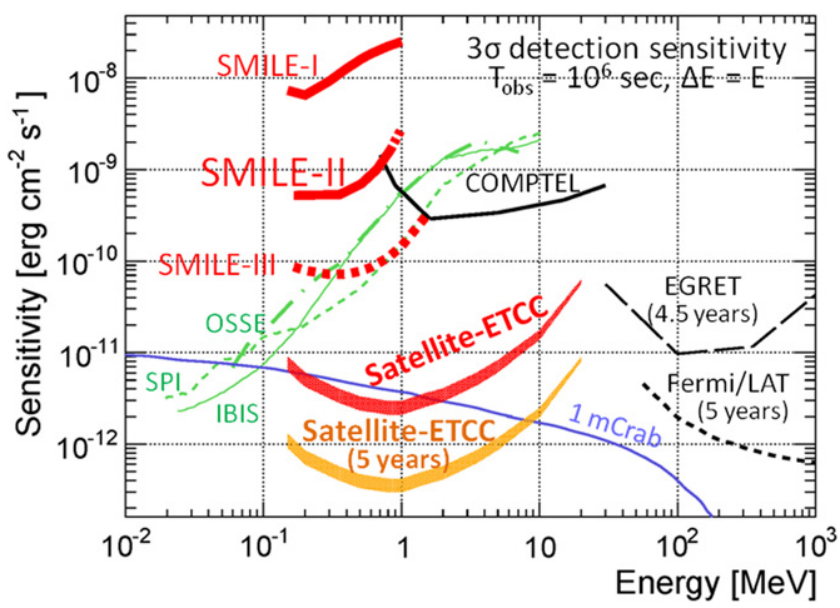

Figure 12. Sensitivities with $3 \sigma$ detection of SMILE-II, III and Satellite-ETCC. Green and black lines are from previous results (Atwood et al. 2009; Takahashi et al. 2013).

the target position, whereas they exude sparsely over the FOV in conventional CC. With this, $\sim 10$ times better sensitivity than CC per unit effective area will be certainly expected (Figure 9). Also, the radical reduction of almost all the instrumental background is found to be attainable by the use of the measured $d E / d x$ of the recoil electron, a kinematical test of the $\alpha$ angle and the optimization of the scattering material such as $\mathrm{CF}_{4}$. Our ETCC has changed delicate CCs to a very tough and reliable gamma imaging device under intense radiation conditions, notably the space. Thus, by resolving two serious problems of the $\mathrm{MeV}$ gamma-ray astronomical observations, we have reached the intrinsic sensitivity determined by its effective area, PSF, and the background of cosmic diffuse gammas, as follows. Figure 12 shows the expected sensitivities of the SMILE balloon experiments, along with a satellite-ETCC consisting of four $50 \mathrm{~cm}$-cubic ETCCs, which, with its large FOV, provides the sensitivity of $\sim 2 \times 10^{-12} \mathrm{erg} \mathrm{cm}^{-2} \mathrm{~s}^{-1}$ at $1 \mathrm{MeV}$ for $10^{6} \mathrm{~s}$ observation, and $\sim 3 \times 10^{-13} \mathrm{erg} \mathrm{cm}^{-2} \mathrm{~s}^{-1}$ for continuum gammas with a sensitivity of $\sim 10^{-7}$ gamma $\mathrm{cm}^{-2} \mathrm{~s}^{-1}$ for line gamma-rays in 5 years operation ( $>100$ times better than those of COMPTEL in 9 years), where a duty factor of 0.5 in the operation is assumed. Note that the green and black lines are from the previous results (Atwood et al. 2009; Takahashi et al. 2013). The background observed in SMILE-I was used for the SMILE-II and SMILE-III simulations. For the background for the Satellite-ETCC, we used the value twice as the extragalactic diffuse gamma flux in $0.1-5 \mathrm{MeV}$ reported with SMM (Watanabe et al. 1999) and COMPTEL (Weidenspointner et al. 2000), assuming the instrumental background to be at the same level as the extragalactic diffuse gamma. The PSF $(\theta=1.2)$ with the ARM and SPD resolutions of $2^{\circ}$ and $5^{\circ}$ was used for the SatelliteETCC, and the PSF $\left(4^{\circ}\right)$ with the ARM and SPD resolutions of $5^{\circ}$ and $25^{\circ}$ was used for the SMILE-II and III. One half of the detected gamma rays from a point source were used in the calculations of the sensitivity. The application of ML-EM to the ETCCs may improve the sensitivities by several factors. However, it is not essential because we successfully provide, for the first time, quantitatively reliable sensitivities for CCs without the use of an optimization algorithm like the ML-EM 
method. Thus, satellite-ETCC would access the deepest universe in high energy astronomy.

Furthermore, with its excellent sensitivity it would detect $\sim 15 \mathrm{SNe} \mathrm{Ia} /$ year at distances up to $60 \mathrm{Mpc}$ with required statistical accuracy (Section 1). For the distances up to $100 \mathrm{Mpc}, \sim 500$ and nearly a thousand $\mathrm{SNe} / 5$ years $(\sim 5 \sigma$ in 150 days) would be expected with and without optical coincidence, respectively, with no observational bias, thanks to the large FOV and high transparency of the MeV gammas. Note that this sample should include some collapsars, in which production of ${ }^{56} \mathrm{Ni}$ is considered to be less than that in $\mathrm{SN}$ Ia.

Also, the well-defined reconstruction of Compton events with low background endows the ETCC with an excellent polarimetry (simulated modulation factors of 0.6 and 0.5 at 200 and $500 \mathrm{keV}$, respectively). The Satellite-ETCC would measure $\sim 10 \%$ polarization $(3 \sigma)$ for $20 \mathrm{mCrab}$. Thus, such observations would open a new era of astronomy with distinctive detections of thousands of $\mathrm{MeV}$ objects.

We thank very much Prof. Keiichi Maeda at Kyoto University and Dr. Alexander Summa at Wurzburg University for the fruitful discussions about $\mathrm{SNe}$. This study was supported by the Japan Society for the Promotion of Science (JSPS) Grant-in-Aid for Scientific Research (S) (21224005), (A) (20244026), JSPS Grant-in-Aid for Challenging Exploratory Research $(23654067,25610042)$, a Grant-in-Aid from the Global COE program "Next Generation Physics, Spun from Universality and Emergence" from the Ministry of Education, Culture, Sports, Science and Technology (MEXT) of Japan, and Grant-in-Aid for JSPS Fellows (09J01029, 11J00606, 13J01213). Also, this study was supported by the joint research program of the Solar-Terrestrial Environment Laboratory, Nagoya University and the National Institute of Polar Research through General Collaboration Projects No. 23-3. Some of the electronics development was supported by KEK-DTP and Open-It Consortium. Finally, we thank the anonymous referee for carefully reading the manuscript and providing helpful comments to improve it.

\section{REFERENCES}

Agostinelli, S., Allison, J., Amako, K., et al. 2003, NIMPA, 506, 250 Aprile, E., Curioni, A., Giboni, K. L., et al. 2004, NewAR, 48, 257 Attwood, D., Bell, P., Bull, S., et al. 2006, NIMPB, 251, 41 Atwood, W. B., Abdo, A. A., Ackermann, M., et al. 2009, ApJ, 697, 1071
Bandstra, M. S., Bellm, E. C., Boggs, S. E., et al. 2011, ApJ, 738, 8 Biagi, S. F. 1999, NIMPA, 421, 234

Boggs, S. E. 2006, NewAR, 50, 604

Chevalier, R. A. 1992, Natur, 355, 691

Churazov, E., Sunyaev, R., Isern, J., et al. 2014, Natur, 512, 406

Greiner, J., Iyudin, A., Kanbach, G., et al. 2009, ExA, 23, 91

Hillebrandt, W., \& Niemeyer, J. C. 2000, ARA\&A, 38, 191

Inoue, Y., Murase, K., Madejski, G. M., \& Uchiyama, Y. 2013, ApJ, 776, 33

Isobe, T., Hamagaki, H., Ozawa, K., et al. 2003, in Nucl. Sci. Symp. Conf. Rec. 2003 IEEE, 5, 3652

Iyudin, A. F., Diehl, R., Bloemen, H., et al. 1994, A\&A, 284, L1

Kabuki, S., Kimura, H., Amano, H., et al. 2010, NIMPA, 623, 606

Kanbach, G., Andritschke, R., Zoglauer, A., et al. 2006, NIMPA, 541, 310

Kurosawa, S., Kubo, H., Hattori, K., et al. 2010, NIMPA, 623, 249

Maeda, K., Terada, Y., Kasen, D., et al. 2012, ApJ, 760, 54

Maoz, D., \& Mannucci, F. 2012, PASA, 29, 447

Matsuoka, Y., Tanimori, T., Kubo, H., et al. 2015, JInst, 10, C01053

Matz, S. M., Share, G. H., Leising, M. D., Chupp, E. L., \& Vestrand, W. T. 1988, Natur, 331, 416

Mészáros, P., \& Rees, M. J. 2010, ApJ, 715, 967

Miuchi, K., Nishimura, H., Hattori, K., et al. 2010, PhLB, 686, 11

Mizumura, Y., Tanimori, T., Kubo, H., et al. 2014, JInst, 9, C05045

Nakauchi, D., Suwa, Y., Sakamoto, T., Kashiyama, K., \& Nakamura, T. 2012, ApJ, 759, 128

Nakazawa, K., Takahashi, T., Ichinohe, Y., et al. 2012, Proc. SPIE, 8443, $84430 \mathrm{E}$

Orito, R., Kubo, H., Miuchi, K., et al. 2004, NIMPA, 525, 107

Sauli, F. 1977, CERN-77-09

Sawano, T., Tanimori, T., Kubo, H., et al. 2014, JPSCP, 1, 013099

Schönfelder, V. 2004, NewAR, 48, 193

Schönfelder, V., Aarts, H., Bennett, K., et al. 1993, ApJS, 86, 657

Schönfelder, V., Bennett, K., Blom, J. J., et al. 2000, A\&AS, 143, 145

Summa, A., Ulyanov, A., Kromer, M., et al. 2013, A\&A, 554, A67

Takada, A., Hattori, K., Kubo, H., et al. 2005, NIMPA, 546, 258

Takada, A., Kubo, H., Nishimura, H., et al. 2011a, ApJ, 733, 13

Takada, A., Tanimori, T., Kubo, H., et al. 2011b, Proc. of 20th ESA Symp. on European Rocket and Balloon Programmes and Related Research ed. L. Ouwehand (ESA Communications, ESTEC, Noordwijk, The Netherlands), SP-700, 567

Takahashi, T., Uchiyama, Y., \& Stawarz, L. 2013, APh, 43, 142

Tanimori, T., Ikeno, M., Kubo, H., et al. 2012, Proc. SPIE, 8443, 84430D

Tanimori, T., Kubo, H., Miuchi, K., et al. 2004, NewAR, 48, 263

Thun, R. 1988, NIMPA, 273, 157

Vetter, K., Chivers, D., Plimley, B., et al. 2011, NIMPA, 652, 599

von Ballmoos, P., Alvarez, J., Barrière, N., et al. 2012, ExA, 34, 583

Watanabe, K., Hartmann, D. H., Leising, M. D., \& The, L.-S 1999, ApJ, 516,285

Weidenspointner, G., Varendorff, M., Kappadath, S. C., et al. 2000, AIP Conf. Proc., 510, 467

Weidenspointner, G., Varendorff, M., Oberlack, U., et al. 2001, A\&A, 368,347

Wilderman, S. J., Clinthorne, N. H., Fessler, J. A., \& Rogers, W. L. 1998, in Nucl. Sci. Symp. Conf. Rec. 1998 IEEE, 3, 1716

Zhang, P., \& Beacom, J. F. 2004, ApJ, 614, 37 